\title{
THE RELATIONSHIP BETWEEN SYMPTOMATIC ASSESSMENT AND CLINICAL SIGNS OF DRYNESS IN DRY EYE SUBJECTS
}

\author{
Mohd Hafidz Ithnin ${ }^{1}$, Azrin Esmady Ariffin ${ }^{2}$ and Khairidzan Mohd Kamal ${ }^{3}$ \\ ${ }^{1}$ Department of Optometry and Visual Science, Kulliyyah of Allied Health Sciences, \\ International Islamic University Malaysia, Pahang, Malaysia, ${ }^{2}$ Faculty of Optometry \\ and Visual Science, Segi University, Selangor, Malaysia, ${ }^{3}$ Department of \\ Ophthalmology, Kulliyyah of Medicine, International Islamic University Malaysia, \\ Pahang, Malaysia.
}

Presenter: Mohd Hafidz Ithnin, mohdhafidz_ithnin@iium.edu.my

Introduction: There was no previous study to assess relationship between dry eye symptoms and signs in the local population. The purpose of this study is to evaluate the relationship of symptomatic assessment and clinical signs of dryness in dry eye subjects.

Materials and method: A Cross-sectional study with convenient sampling involving 321 subjects with 642 eyes was done. Subjects were divided into non-dry eye (NDE) and dry eye (DE) groups. The division of NDE and DE groups were done using two types of classification. In Classification 1 (symptoms-based), DE was defined if the score of Ocular Surface Disease Index (OSDI) score was more than 33. The value of tear film break-up time (TFBUT) less than 5 seconds and Schirmer test with anaesthesia (STA) less than $5 \mathrm{~mm} / 5$ minutes were considered as DE for Classification 2 (signs based).

Results: There were poor correlations between OSDI and clinical signs of dryness in all subjects involved $(p>0.05)$. Similar findings were also noted within NDE subjects in Classification 1 and Classification 2. OSDI score was also not significantly correlated with the signs of dryness within DE subjects except with ocular protection index (OPI) ( $r=$ $0.14, p=0.047$ ) in Classification 1 and with conjunctival lissamine green staining (CLGS) $(r=0.23, p=0.040)$ in Classification 2 .

Conclusion: There were no significant correlation between the symptoms of dry eye and the clinical signs of dry eye. Therefore, the clinical diagnosis of dry eye can be made based on either just on the symptoms or the signs. 